
illustrations to Arveen Bajaj at the BDJ, 64 Wimpole Street, London W1G 8YS. Trade news is supplied as a service to the reader and does not imply endorsement by the BDJ. Normal and prudent research should be exercised before purchase of use of any product mentioned.

\section{Periodontal hygiene}

The new Gi-Mask Automix gives precise reproduction of the gingival soft tissue, interdental spaces and papilla helping to provide ideal conditions for periodontal hygiene and aesthetics, according to its manufacturer.

It is fast and easy to use and is available in $75 \mathrm{ml}$ cartridges, fitting conventional dispensers, and features a 'low waste' mix tip for economy.

Reader response number 50

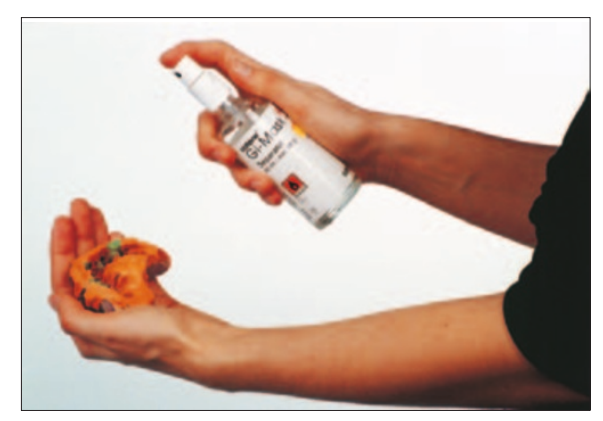

Making your mark

The toothbrush handle imprinting service from Oral-B allows dentists to order the Oral-B CrossAction toothbrush with a message of their choice printed on the handle.

Practices can print a message of up to sixty-six characters and one of three logos - an apple, a tooth or a smiling face allowing dentists to add individuality to a toothbrush and ensure their patients are reminded of them each time they brush their teeth.

Reader response number 52

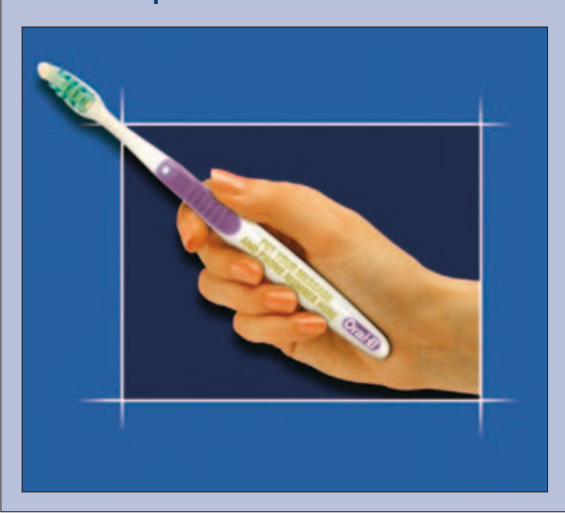

\section{New light-curing composite launched}

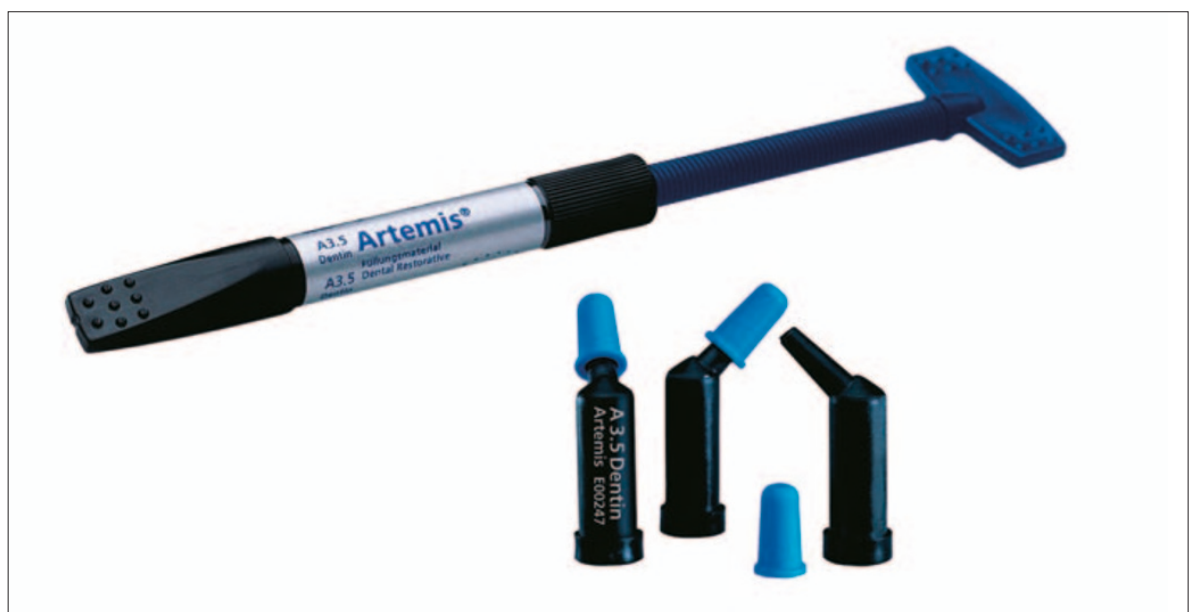

Artemis from Ivoclar Vivadent is a new light-curing composite. The wide range of dentine, enamel and effect shades in various degrees of translucency allow the natural appearance of teeth to be restored.

Artemis can be used for a wide range of indications for both anterior and posterior teeth. The system includes 30 shades in various degrees of translucency and the quality shade guide features ceramic samples ensuring consistent shade selection results as a basis for highly aesthetic

restorations. The product is characterised by modelling properties, high stability and shade fidelity, and is easy to polish to a high gloss.

The material's physical properties, such as radiopacity and low light sensitivity, allows the processing time to be extended under optimal light conditions.

It is supplied in syringes as well as in Cavifils, both of which are available in 30 shades.

Reader response number 51

\section{Interchangeable tips in Flexichange range}

The Flexichange range, made in the UK by Dentsply Ash has interchangeable tips which require only worn points to be replaced rather than the entire instrument.

The product is available in 49 periodontal instruments, so there is a wide array of tips to choose from. The design stage of the Flexichange range assigned the highest priority to the adhesion of the silicone to the steel inner tube and therefore the whole length of the silicone is bonded to the inner tube which can significantly reduce the risk of cross-infection. A quality stainless steel is used and the tips are toughened and tempered to keep cutting edges sharper for longer.

These ultra-sharp edges also help to reduce repetitive cutting strokes and increase efficiency according to the company. A raised dot pattern on the handles has been specially designed to provide a non-slip grip.

Flexichange comes in six colours dark lilac, light blue, deep pink, sun yellow, leaf green and vibrant orange. Reader response number 53

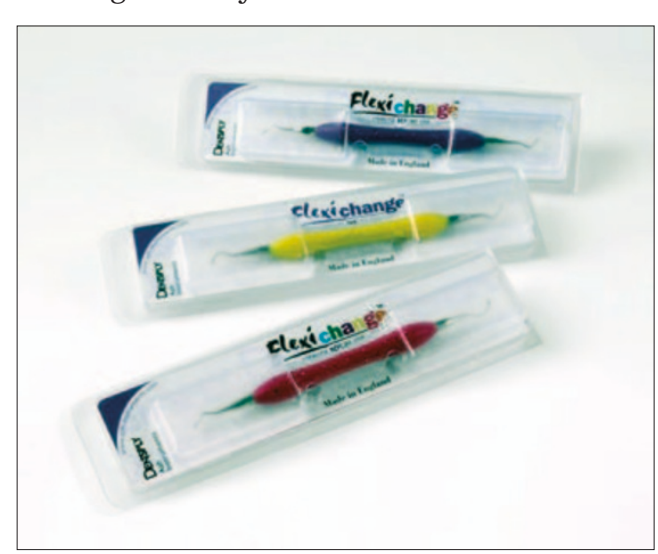




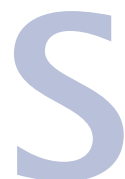

\section{Dust free alginate}

The Kent Alginate Extra is a fast setting, dust free Alginate (Class A Type 1) with colour indication.

The material sets in the mouth in 60 seconds and the total setting time (including working time) is 2 minutes 30 seconds.

Reader

response

number 54

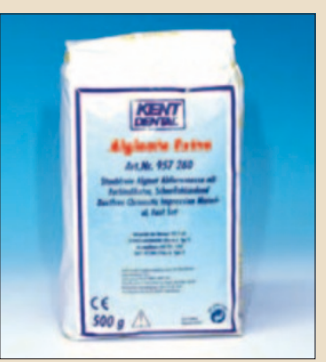

\section{Temporary crowns}

Henry Schein offers a wide selection of temporary crown materials to address aspects of temporary crown and bridge work such as Polycarbonate Temporary Crowns from Directa, 3M ESPE and Henry Schein. Polycarbonate crown forms from Henry Schein are easily scissor-trimmed, contoured, crimped and polished and their thin design prevents gingival irritation.

Its Silver Tin Temporary Crowns are made from high purity tin alloy, which is soft, non-galvanic and tasteless and the Stainless Steel Temporary Crowns are available in two different types for primary molars or permanent crown replacement.

Finally, the Aluminium Temporary Crowns from Henry Schein, Myerson Ltd and Austenal KRI Aluminium Preformed Crowns are presented in a pack of 150 assorted preformed crown sizes.

Reader response number 55

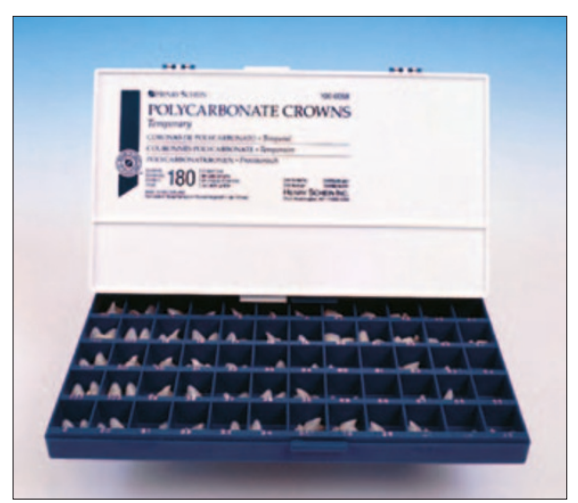

\section{New silicone impression material}

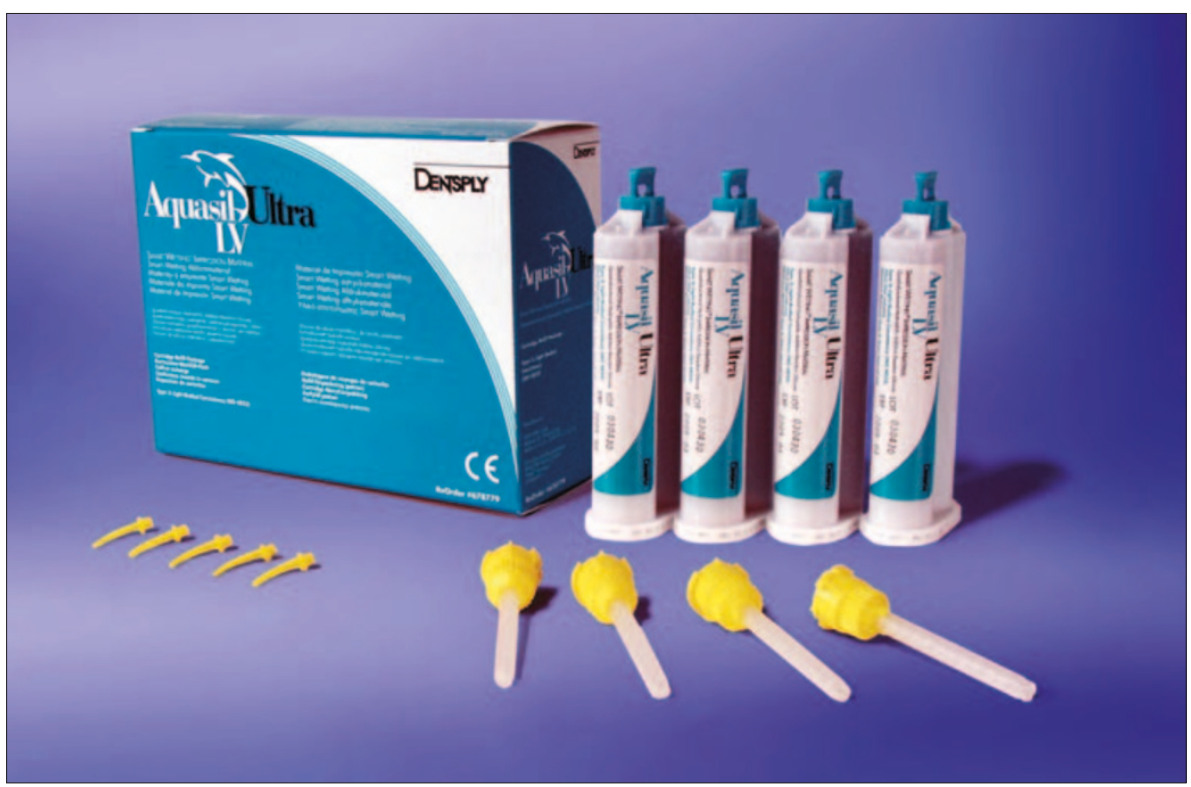

Aquasil Ultra, the new addition silicone impression material from Dentsply has outstanding 'wetability', accuracy and resolution and tear strength according to the company.

Its tear strength and dimensional stability ensures that resolution is maintained, even after multiple stone pours.

The combination of ultra 'wetability' and strength ensures that even the thinnest margins are recorded and will remain intact when the impression is removed from the mouth.

The product is designed to minimise the impact of voids, tears, bubbles, pulls and drags. It is available in LV wash (fast and regular set) and XLV wash (fast and regular set) formulations as well as Heavy and Monophase in the DECA format.

Reader response number 56

\section{Wide range of glass ionomers}

Henry Schein's extensive glass ionomer range offers a wide variety of both new and well established glass ionomers from leading manufacturers to suit all requirements according to the company. This includes ChemFlex from Dentsply, Diamond Carve from Associated Dental, Fuji IX from GC UK, Hi-Fi from Shof and Voco Ionofil from Voco.

Also available are the Ketac, Photac Fil and Vitremer ranges from $3 \mathrm{~m}$ Espe, l-Vitro Eleven and VLC from Panadent and RGI, Henry Schein's own brand.

Reader response number 57

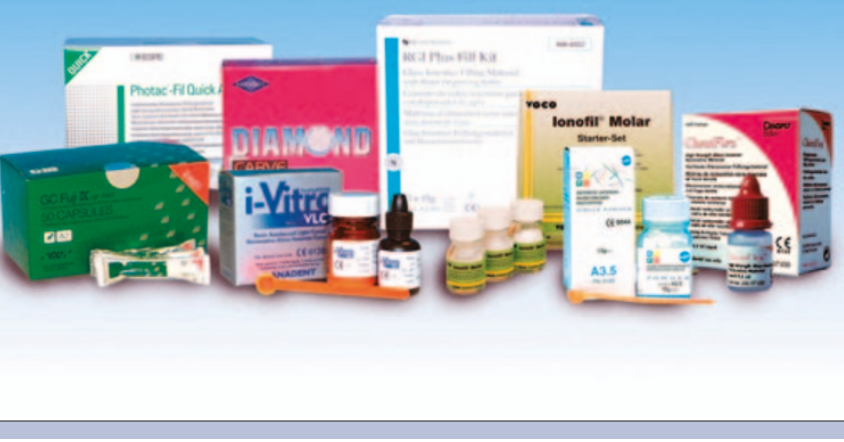




\section{New veneering material launched}

VITA has introduced a new veneering material for dental technicians who intend to prepare high-quality all ceramic restorations in a highly efficient and economic manner.

VITAVM 7 protects opposing teeth and is suitable for veneering all ceramic framework materials in the CTE range of approximately 7.2 to 7.9 , such as VITA InCeram Alumina, Lumina, Spinell and Zirconia.

It features a new logically arranged shade and layering concept which allows the user to achieve intensive or translucent shade effects with one veneering ceramic. They can select between basic layering consisting of two materials and build-up layering which includes the application of three materials.

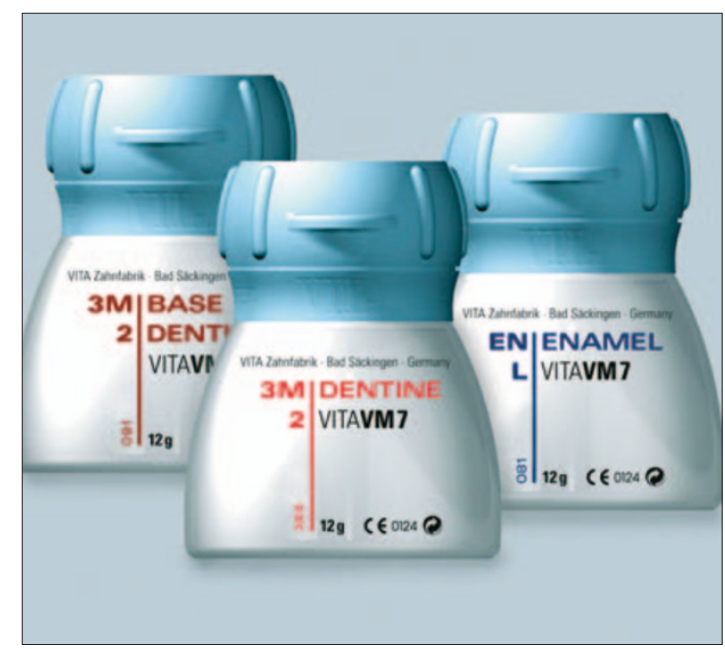

According to the company it excels by

its very low chemical solubility and thermal shock resistance between ceramic framework and ceramic and superior flexural strength.

Reader response number 58

\section{Bonding system with nanofiller}

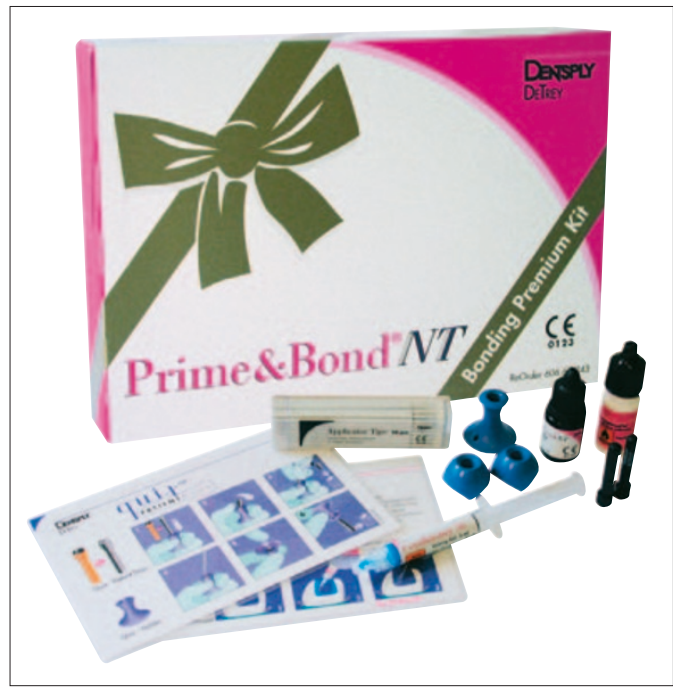

Prime \&t Bond NT all-purpose, selfpriming dentine/enamel bonding system from Dentsply has a unique nanofiller added to the formulation.

It features higher bond strength, reduced technique sensitivity, a oneapplication procedure and reliable and consistent results according to the firm.

In addition to this, Prime \& Bond NT Quix is a single unit dose delivery system, with convenience in mind.

An ergonomic holder also keeps the container in an upright position and enables the tip to snap off easily, thus reducing the risk of crosscontamination.

Reader response number 59

\section{Suitable for temporary crowns and bridges}

UnoTemp SC from The Dental Directory is suitable for inlays and onlays as well as temporary crowns and bridges.

It offers the user rapid processing with its tri-phase polymerisation (30-60 seconds in the mouth) and can aid patient comfort and acceptability as well as practitioner usage.

For example, little heat is emitted during polymerisation to minimise discomfort and as it is MMA free it has a neutral taste and odour.

It is available in three of the most popular Vita shades can be highly polished, and has

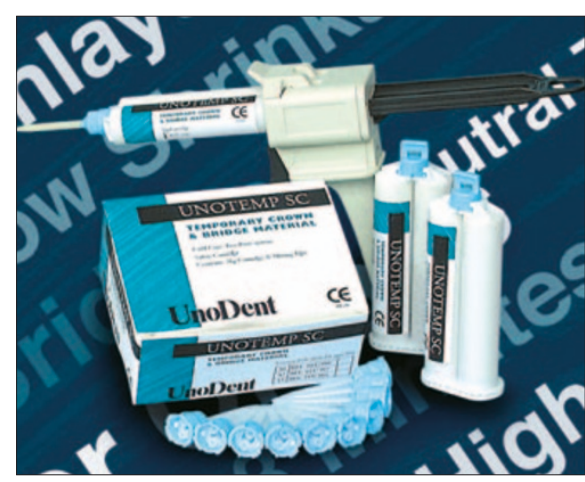
low water uptake thus maintaining a high level of colour stability according to the company. UnoTemp SC is part of The Dental Directory's own brand of products called UnoDent.

Reader response number 60 